\title{
Exploring Promising Concepts and Critical Technologies for Offshore Methane Hydrate Development System
}

\author{
Yo MATSUDAIRA ${ }^{\mathrm{a}}$, Ryota WADA ${ }^{\mathrm{a}, 1}$, Yoshihiro KONNO ${ }^{\mathrm{a}}$, \\ Go HASHIMOTO ${ }^{\mathrm{b}}$ and Ken TAKAGI ${ }^{\mathrm{a}}$ \\ ${ }^{a}$ Graduate School of Frontier Science, The University of Tokyo, Japan \\ ${ }^{\mathrm{b}}$ Faculty of Engineering, The University of Tokyo, Japan
}

\begin{abstract}
Natural gas production from offshore methane hydrates can increase the energy security conditions for many countries, such as Japan. Methane hydrate is an unconventional source of carbon hydrates and further research is required to achieve cost efficient production. However, the concept of development system, such as production facilities, has yet been decided. Production facility system comprise many equipment and technology, and it is not easy to decide or prioritize which technology to invest research resource in. The problem requires an architectural decision under technological uncertainty. In this paper, we follow the systems approach to evaluate various possibilities of production facility system and measure the interactions that occur between each technology development and architectural decisions. In the course of the study, we utilize domain knowledge such as analogy to existing offshore gas production facilities to define system functions, and existing R\&D projects to consider TRL of each technology component. Gathering expert's knowledge was crucial to tackle such multidiscipline problem. The important index for concept selection was flexibility and mobility considering the uncertainty of well production lifetime. While optimum concept varies among the various field conditions, we find some technology that are critical in many promising concepts. Finally, we propose critical technology sets and several promising concepts, together with discussion on the role of system architecture in such problem.
\end{abstract}

Keywords. Methane Hydrate, Production Facility System, Systems Approach, Promising Concepts, R\&D Investment

\section{Introduction}

Methane hydrate is an ice-like compound containing methane gas [1]. With abundant resource potential, many countries are eager to develop this untapped source of carbon hydrate. Since accumulation of methane hydrates often occurs in regions different from conventional oil and gas fields, countries nearby are highly motivated in its development to increase self-sufficiency of energy. Significant accumulations have been identified on the North Slope of Alaska, USA; in the Northwest Territories of Canada; in the Gulf of Mexico; and offshore Japan, India, South Korea and China [2].

\footnotetext{
${ }^{1}$ Corresponding Author, Email: r_wada@k.u-tokyo.ac.jp.
} 
Japan aims to establish methane hydrate development technologies and transfer them to private oil entities through "Japan's Methane Hydrate R\&D Program" [3]. Japan will carry out technology development during the period from 2023 to 2027 with the aim of projects for commercialization led by private sector companies being commenced." (Strategic Energy Plan, 2018). However, the development stage is still at its early stage, still far from commercial development stage in terms of technical, economic and social conditions. One of the largest differences between production of methane hydrate and conventional oil and gas is that, oil and gas flow out of the reservoir with its high pressure, where methane hydrate needs to be dissociated for the production. "Depressurization method-based approach" is considered as the most promising approach to dissociate hydrates [4]. In addition, many of the methane hydrate layers exist in unconsolidated layers that can induce productivity reduction. As this remains the largest concern, the main issue of $R \& D$ is to demonstrate a continuous production method from hydrate reservoirs.

Despite many studies on the production process of methane hydrates, the production facility system, connecting the wellhead to onshore facility, has not gained much attention. Production system will share a lot of existing technologies developed in the offshore oil \& gas fields. However, some modifications are required to meet the characteristics of methane hydrates. The production phase requires higher cost, and it is crucial to decrease the cost of production facility for $\mathrm{MH}$ development to be cost competitive. Further R\&D is essential for the commercialization of $\mathrm{MH}$, and it must be done now to meet the ambition.

Production facility system comprise many equipment and technology. However, we do not know which system architecture of the production facility is promising. Production facility system requires expertise of various disciplines, such as petroleum engineering, ocean engineering, subsea engineering and so on. The complex interaction of equipment and technology makes the concept selection and defining the critical technology identification difficult. Thus, it is not easy to decide or prioritize which technology R\&D to invest in.

On the contrary, a typical concept selection for offshore systems is often based on track records of existing projects. The large risk of offshore oil \& gas projects makes the industry rather risk adverse. Buckley and Uehara [5] conducted a screening assessment of multiple subsea development concept alternatives for typical Brazilian pre-salt deep water applications. They chose several competitive concepts based on cost, application, criteria, premises and field characteristics. This served as a guidance of concept selection. Although we see the analogy in concept level selection, the analysis is comparison existing technology and not directly applicable to new concept development.

The outstanding question of this paper is "What R\&D topic to invest in for the commercialization of $M H$ ?". MH development is a new system, where the good system architecture is still unknown. Unless the system architecture is not decided, we cannot prioritize which technology to invest research resource in. On the contrary, innovative future concepts can give driving force to $R \& D$ investment.

The problem can be viewed as a technology decision under architectural uncertainty for methane hydrate development. Since the architecture of production system has a wide range of available architectures, its interpretation can be difficult. Battat et al [6], proposed a method to evaluate individual technologies within the tradespace and measure the interactions that occur between technologies influencing architectures across a wide tradespace. Hiekata et al [7], compared the importance of 
evolving IoT technology in shipping industry. The evaluation was based on systems approach, combining several sub-system models to simulate the complex interaction of each technology development on the QCD (Quality, Cost and Delivery) of shipping. A preliminary report on the overall cost for gas production from methane hydrate production has been studied by Walsh et al. [8], where the production facility was set similar to typical offshore gas production system.

The aim of this paper is to answer the outstanding question through systems approach, which will be briefly described in Section 1. The description of $\mathrm{MH}$ production facility system is given based on systems approach in Section 2. Section 3 illustrates the analysis results, which is further discussed in Section 4. A brief conclusion is provided at the end.

\section{Methodology}

\subsection{Why systems approach?}

The difficulty of analyzing MH production facility system comes from the complexity and socio-technical uncertainty. Production facility system requires expertise of wide discipline, such as petroleum engineering, ocean engineering, subsea engineering and so on. The complex interaction of equipment and technology makes the problem difficult to tackle by a single expert. In addition, the problem has many stakeholders, ranging from government officials to local communities. It is inevitable to consider social aspects in the decision-making process.

Since the system is new, we must be open-minded to include variety of concept, i.e. broad solution space. An exhaustive approach to employ creativity is desired. Since our aim is to evaluate various system architecture performance in the near future, technical, social and market conditions at that time are uncertain. In the evaluation of each system, we must incorporate such uncertainty.

The final aim is to provide information to the stakeholders to make good decisions. There are various stakeholders of the $\mathrm{MH}$ project, and the consensus needs to be based on objective evaluation. The stakeholders need clear understanding of how the information was constructed, i.e. the process towards finding the answer to the question needs to be transparent and clear. It is impossible to have detailed design for each concept at this stage, but we must provide information with adequate granularity.

To meet these requirements, we need to reduce ambiguity, employ creativity and manage complexity. System architecture is the study of early decision making in complex systems [9] and provides the approach to answer the problems above.

\subsection{Brief introduction of systems approach}

In systems approach, the complex problem is decomposed into several parts. First, stakeholder value network analysis is conducted to identify the important performance of the system. Next, we analyze existing systems to describe the system as a collection of intent, function and form. We seek for ideas to improve each component of the system to extract wide variety of possible concepts. Finally, each concept is evaluated and projected on tradespace. Throughout the process, we aim to reduce ambiguity, enhance creativity, and manage complexity. 
The scope of this paper is to evaluate $\mathrm{MH}$ production facility system for commercialization in the future. Thus, the paper will focus on the system architecture, which is the initial stage for systems approach. In this paper, we consider stakeholder value network analysis, define system boundary and consider a morphological matrix.

\section{System description}

\subsection{Stakeholder value network}

The stakeholder value network (SVN) for MH production facility system for the situation in Japan is depicted in Figure 1. The SVN indicates two main value that drive the decision of each stakeholder network. The first is the economic competitiveness, where the cost of the produced natural gas needs to be cost effective compared to other source of power generation. The other is on risk of the production system. The risk can be separated into two aspects, one is risk that leads to loss of production, and the other is risk that has environmental impact. The former can be considered as economic impact, where the latter has larger impact on wider stakeholders.

In this study, we set the two main value specification of the project as cost and risk. Since these two values cause tradeoff in between each other, it is up to the decision maker's, or the stakeholders', consensus to choose the best balance between them.

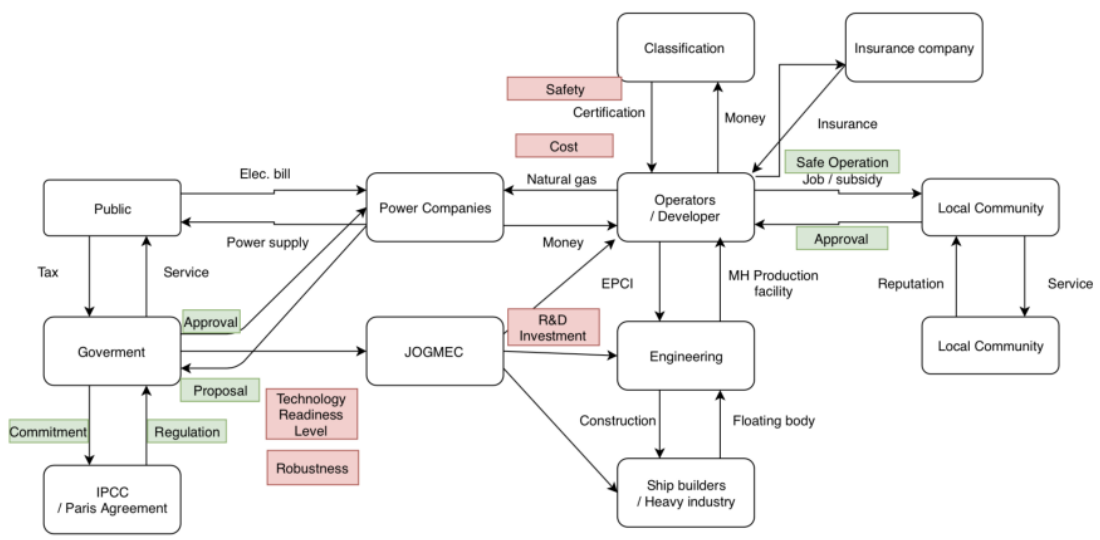

Figure1. Stakeholder value network for $\mathrm{MH}$ development.

\subsection{Scope and system boundary}

The scope of this paper is to evaluate $\mathrm{MH}$ production facility system for commercialization in the future. Thus, we will consider fields that has large enough reservoir for such scale. The technology can include ones that are currently under R\&D. The field condition is depicted in Table 1. Production fluid setting is taken from [10].

Table 1. Equipment placement options for morphological matrix.

\begin{tabular}{cccc}
\hline Water depth & Field size & MH layer thickness & Production period \\
\hline $1,500 \mathrm{~m}$ & $30 \mathrm{~km}^{2}$ & $200 \mathrm{~m}$ & 30 years \\
\hline
\end{tabular}


In this paper, the system boundary is from the wellhead to onshore facility. The production process before the wellhead is currently being investigated, but we consider the input into our system boundary can be assumed in several conditions. The output of the system is limited to natural gas into onshore facility.

\subsection{Inference from existing offshore oil \& gas systems}

As mentioned before, the production facility system for MH shares a lot of features with that of existing offshore oil \& gas production. We first analyzed the common and unique features among both systems $[11,12,13]$.

The sample of methane hydrate taken from the Nankai Trough suggests that more than $99.9 \%$ of the gas included in the methane hydrate is methane and other hydrocarbons heavier than ethane are less than $0.01 \%$. This eliminates the need for processing heavy hydrocarbons and other contaminants such as acid gas.

Since the natural gas is expected to be produced by depressurization process, the gas will have lower temperature and lower pressure compared to existing oil \& gas fields. Since dehydration is processed under low temperature and high pressure, a compression process is necessary before dehydration. However, the gas needs to be compressed anyway for pipeline transportation, so the necessary equipment is basically the same.

Figure 2 shows the interaction of equipment that consist the MH production facility system. The interaction suggests that the equipment can be clustered into several groups based on function and their interactions.

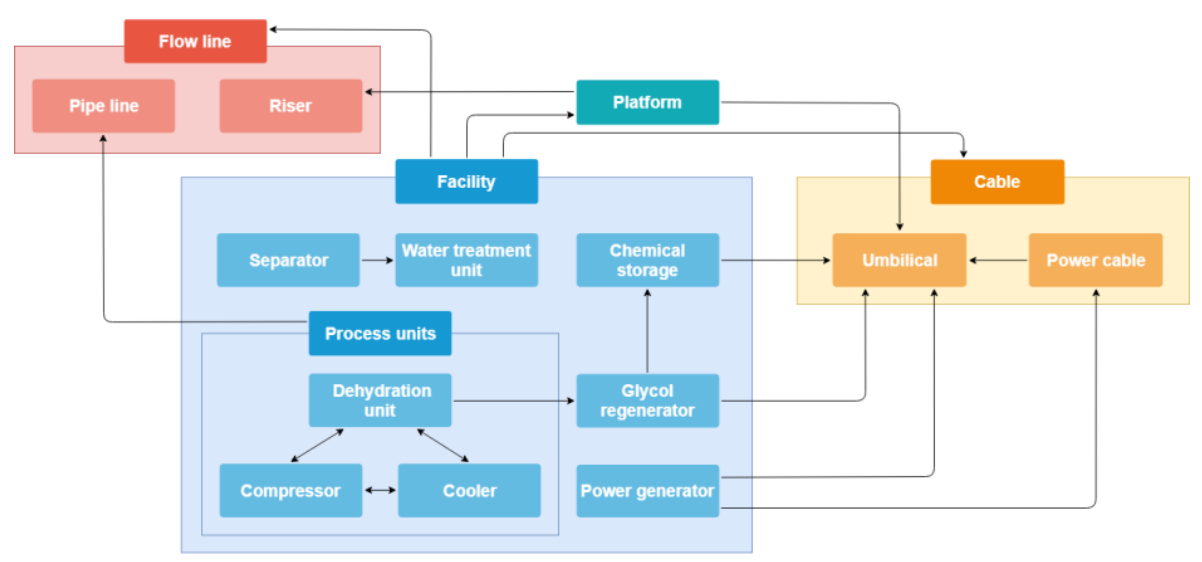

Figure 2. Relation of equipment that consist the $\mathrm{MH}$ production facility system.

\subsection{Morphological matrix}

The choice of concepts can be exhaustively considered by applying a morphological matrix. The matrix comprises of options we have. In this context, the concept is characterized by the location of each component.

In Table 2, we have listed the location options for the equipment. First, the location is listed without considering the current level of technology. The technologies 
under development, such as subsea glycol regenerator, would require further R\&D and be evaluated with handicaps, i.e. higher cost and higher risk. If the concept turns out to be still competitive, that suggests the technology is important.

Table 2. Equipment placement options for morphological matrix.

\begin{tabular}{ccc}
\hline Equipment & \multicolumn{1}{c}{ Location } & Removable \\
\hline Separator & Offshore / Subsea & - \\
Water treatment & Offshore & Yes \\
Compression & Onshore / Offshore / Subsea & \\
Cooling & Onshore / Offshore / Subsea & \\
Dehydration & Onshore / Offshore / Subsea & Yes \\
Glycol regenerator & Onshore / Offshore / Subsea & - \\
Power unit & Onshore / Offshore / Subsea & - \\
Chemical storage tank & Onshore / Offshore / Subsea & \\
\hline
\end{tabular}

From the morphological matrix, we extract 42 concepts. Some concepts were screened out considering technical feasibility. These concepts can be considered to cover all the feasible architectural space of $\mathrm{MH}$ development systems.

\section{Tradespace analysis}

\subsection{System of systems model}

The cost and risk of production facility system is evaluated under several scenarios, taking technical feasibility into account. A model to evaluate such broad perspective requires system of systems model, combining system models to evaluate each aspect of the whole system.

The scenario model considers the field condition, which here is given as three scenarios with different distance from production field to onshore facility. We do not know how the field conditions will be at this moment, so the uncertainty is evaluated by assuming several scenarios. In the future, scenarios such as period of production and the distance to the next production field can be incorporated.

Technical feasibility of production facility is based on flow assurance and offshore engineering. An example of flow assurance is preventing plugging by hydrate formation or diameter design subsea flowlines. Offshore engineering will consider the installation of pipelines. This is where domain knowledge is crucial. We had private communication with several experts, evaluating flow assurance and offshore engineering.

The cost evaluation is based on the net present value of 30 years project lifetime. Capital expenditure (CAPEX) includes equipment cost, floating body cost, and flowline cost. The cost assumption method is based on a reference cost and factors to consider allocation of the equipment, scale factor, and technical readiness level. For example, equipment located offshore will cost twice the ones onshore, and ones located subsea will be evaluated as triple of the ones onshore. Operating expenditure (OPEX) is estimated from the total CAPEX. Some scenarios include relocation of the production system. Here, the cost learning curve, such as FOAK or NOAK, is not considered. The risk model is based on failure risk of equipment and flow assurance. 
Failure risk depends on TRL and where the equipment is placed. If the technology is under development, the risk is higher.

The initial models are rough estimates. The granularity of each model will need further investigation, considering its impact on the overall results. In such case, we will need expert evaluation and input to validate and refine the model.

\subsection{Result and sensitivity analysis}

The tradespace analysis was run for several scenarios. The results for reference scenario, where the distance to production field is $50 \mathrm{~km}$ from onshore facility, are shown in Figure 3. Sensitivity analysis was considered for $100 \mathrm{~km}$ distance case, equipment cost, platform cost, electric cable cost (Figures 4-9).

The concepts that form the pareto front are described in Table 3 Concept 6 is not in the pareto front for the reference scenario, but forms the pareto front for several sensitivity analysis. The concept currently suggested by JOGMEC [14] is indicated with the red circle as a reference concept in Figure 3.

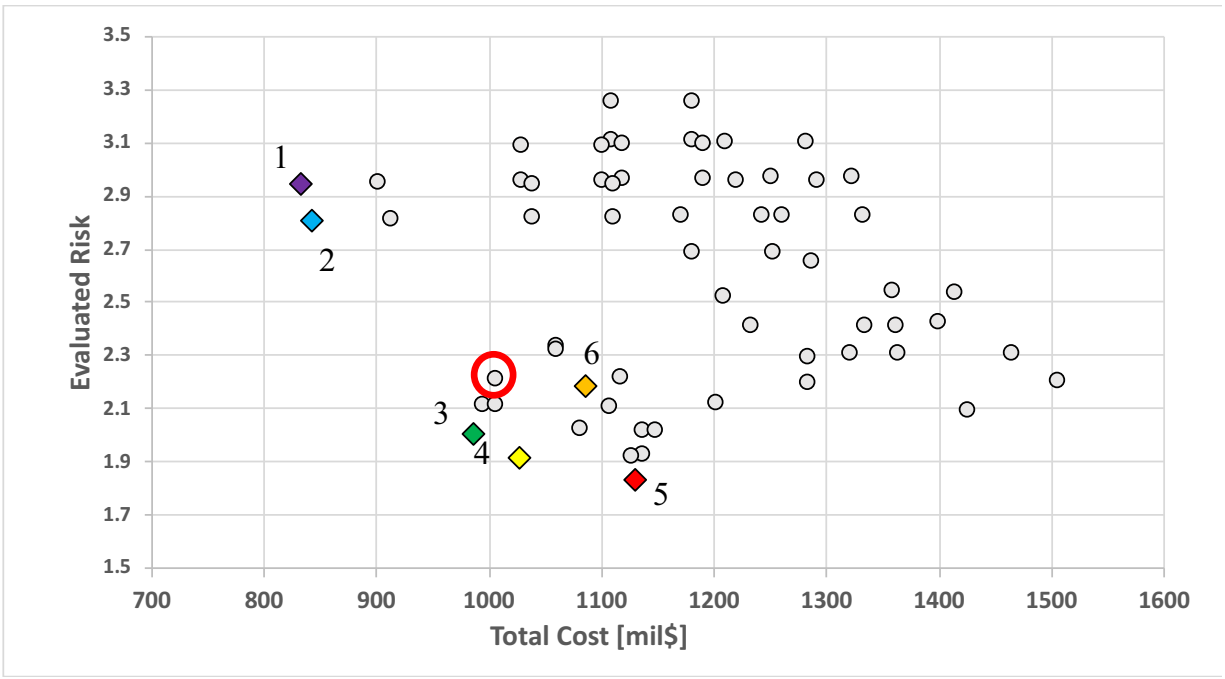

Figure 3. Tradespace results for $100 \mathrm{~km}$.

Table 3 Equipment placement options for morphological matrix.

\begin{tabular}{cccccccc}
\hline Equipment & Concept & $\begin{array}{c}\text { Concept } \\
\mathbf{1}\end{array}$ & $\begin{array}{c}\text { Concept } \\
\mathbf{2}\end{array}$ & $\begin{array}{c}\text { Concept } \\
\mathbf{4}\end{array}$ & $\begin{array}{c}\text { Concept } \\
\mathbf{5}\end{array}$ & $\begin{array}{c}\text { Concept } \\
\mathbf{6}\end{array}$ & $\begin{array}{c}\text { Concept } \\
\text { Ref }\end{array}$ \\
\hline Separator & Subsea & Subsea & Subsea & Subsea & Subsea & Subsea & Subsea \\
Water & None & None & None & None & None & None & Subsea \\
treatment & & & & & & & \\
Compression & Onshore & Onshore & Offshore & Offshore & Offshore & Subsea & Offshore \\
Cooling & Onshore & Onshore & Offshore & Offshore & Offshore & Subsea & Offshore \\
Dehydration & Onshore & Onshore & Offshore & Offshore & Offshore & Subsea & Offshore \\
Glycol & Onshore & None & None & None & None & None & Offshore \\
regenerator & & & & & & & \\
Power unit & Onshore & Onshore & Offshore & Offshore & Onshore & Onshore & Offshore \\
Chemical & Subsea & Subsea & Offshore & Offshore & Offshore & Subsea & Offshore \\
storage tank & & & & & & &
\end{tabular}




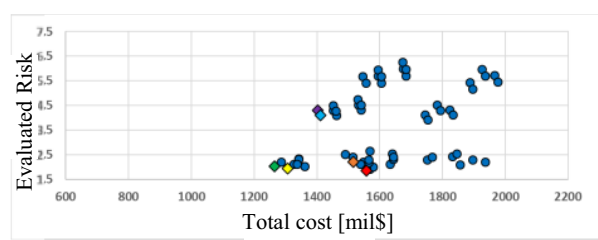

Figure 4. Offshore distance $100 \mathrm{~km}$.

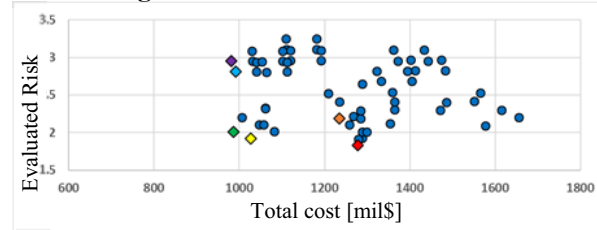

Figure 6. $200 \%$ power cable cost.

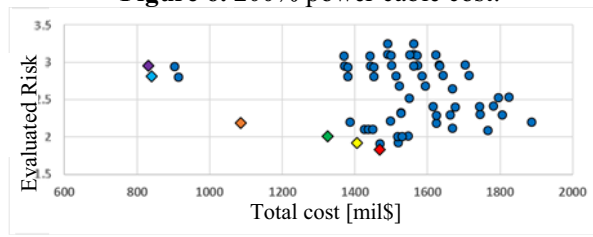

Figure 8. 200\% platform cost.

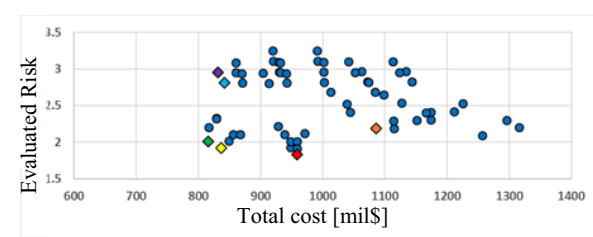

Figure 5. 50\% platform cost.

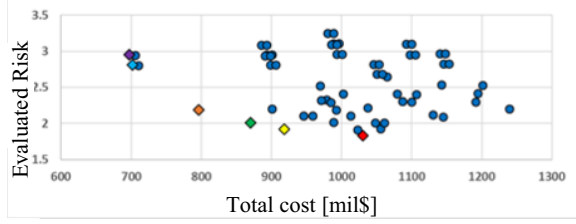

Figure 7. 50\% facility cost.

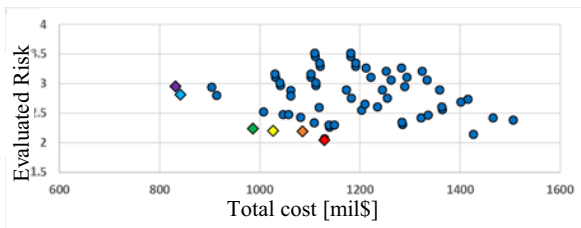

Figure 9. Lower subsea facility risk.

\section{Discussion}

\subsection{Promising concepts}

Tradespace analysis suggests that several concepts comprise the pareto front. Our result agrees well with the concept currently suggested by JOGMEC [14], since the reference concept is located near the pareto front.

From the tradespace analysis for several scenarios, the interesting finding was the promising concepts do not differ greatly. The concepts 1 and 2 are always giving one of the lowest cost systems. This concept is a challenging concept, since the majority of the process is located onshore.

Concepts 3, 4 and 5 share the feature that they utilize a floating body for gas treatment. These concepts resemble the existing offshore oil and gas systems and give lower risk values.

Looking at the different scenarios, the distance from onshore facility has impact on the chosen concepts. This suggests some criteria for prioritizing production fields.

\subsection{Important $R \& D$ topics}

From the results above, we can define important R\&D topics that will have impact on the commercialization of $\mathrm{MH}$ production. We define two fields of important technology, namely fundamental technology area and breakthrough technology area.

The fundamental technology field is defined as the technology which is commonly utilized among the concepts on the pareto front. In our analysis, the separation system 
is located subsea for all 6 concepts. Subsea separation system is a technology that we expect to be critical in the $\mathrm{MH}$ production facility system.

The breakthrough technology field is defined as a technology which is not commonly utilized, but has high potential of improving the performance of some concepts. For example, onshore power generation is considered as competitive in concepts $1,2,5$, and 6 . However, the cost of electric cables is the bottleneck for such technology. Decreasing power cable cost by technology innovation, such as on-site power generation by ocean renewable power generation will provide breakthrough.

For concept 1 and 2, the risk of flow assurance is the concern despite the low cost. This is highly associated with the level of dehydration before the transportation process. Hydration formation occurs in low temperature and high-pressure conditions. Current practice for flow assurance avoids any risk of hydrate formation by utilizing inhibitors and insulation. Otherwise, the gas treatment will require $100 \%$ dehydration. Long distance transportation requires high pressure, however there is an upper limit for pressure to avoid hydrate formation. In such case, boosters need to be equipped subsea. If we allow hydrate formation provided it does not lead to plugging, the constraints for the system design will be relieved. We see high potential of innovation in this area.

\subsection{Systems approach in offshore system design}

The systems approach was demonstrated for the evaluation of $\mathrm{MH}$ production facility system. The analysis presented above is an initial stage evaluation but provided a lot of insights to selection of promising concepts and important R\&D topics. Here, we discuss the effectiveness of systems approach in considering new offshore development systems, like the MH production facility system we discussed in this paper.

Our main goal was to tackle a problem without having bias. We believe this is important for innovation. The function-based decomposition allows us to consider wide variety of concept options through solution neutral thinking. Until the analysis was conducted, onshore gas treatment did not come up to our mind.

The problem is multi-disciplinary, which requires inference from wide variety of experts. Systems approach provides explicit description of the system at each stage, which makes the communication possible with experts. This allows us to continuously refine the model through expert's participation. Such transparency and clarity are also important for the model results to be practical. In many cases, these analyses are only presented with results, which makes it difficult for the decision makers to understand the underlying assumptions. Here, the process is well-defined and organized, which provides accountability to the decision makers. The approach is comprehensible to all during its design, implementation, and evolution.

\section{Conclusion}

We conducted a study based on systems approach to consider "What R\&D topic to invest in for the commercialization of $M H$ ?". 42 concepts that are technically feasible were evaluated. The results showed 6 promising concepts that comprise the pareto front for cost and risk evaluation. From the promising concepts, we identified the 
fundamental technology area and breakthrough technology area for important R\&D topics. These results provide important insights into MH R\&D strategy.

The ocean has a vast potential of resources, which most remain untapped. To utilize them, we need to develop a system from scratch. Our study shows the capability of system approach to provide a methodology to reduce ambiguity, enhance creativity and manage complexity. This is crucial for the co-creation process required to engage many stakeholders and experts from multi-disciplinary fields.

\section{Acknowledgement}

We thank Dr. Kazuo Miura and various experts from industry for providing advice on this research. One of the authors (Y. Konno) would like to thank the Arai Science and Technology Foundation for financial support.

\section{References}

[1] E. Sloan, and C. Koh, Clathrate hydrates of natural gases. CRC press, Boca Raton, 2007.

[2] R. Birchwood, D. Jianchun and D. Shelander, Developments in Gas Hydrates, Oilfield review, 22.1, 18 33., Houston, Texas, USA, 2010.

[3] A. Oyama and S. Masutani, A review of the methane hydrate program in Japan. Energies, 10(10), 1447, 2017.

[4] Y. Konno, T. Fujii, A. Sato and J. Nagao, Key findings of the world's first offshore methane hydrate production test off the coast of Japan: Toward future commercial production. Energy \& Fuels, 31(3), 2607-2616, 2017.

[5] K. Buckley and R. Uehara, Subsea Concept Alternatives for Brazilian Pre-Salt Fields, OTC Brasil. Offshore Technology Conference, 2017.

[6] J. Battat, B. Cameron, A. Rudat and E. Crawley, Technology decisions under architectural uncertainty: informing investment decisions through tradespace exploration. Journal of Spacecraft and Rockets, 51(2), 523-532, 2014.

[7] K. Hiekata, T. Mitsuyuki, R. Ueno, R. Wada and B. Moser, A Study on Decision Support for Introducing IoT Technology to the Maritime Industry. Journal of the Japan Society of Naval Architects and Ocean Engineers, 25, 2017, DOI: 10.2534/jjasnaoe.25.175.

[8] M. Walsh, S. Hancock, S. Wilson, S. Patil and E. Sloan, Preliminary report on the commercial viability of gas production from natural gas hydrates. Energy Economics, 31(5), 815-823, 2009.

[9] E. Crawley, B. Cameron and D. Selva, System architecture: strategy and product development for complex systems. Prentice Hall Press, Harlow, 2016.

[10] M.Kida et al., Chemical and crystallographic characterizations of natural gas hydrates recovered from a production test site in the eastern Nankai Trough, Marine and Petroleum Geology, Volume 66, Part 2, September 2015, pp. 396-403, 2015.

[11] H. Konishi and K. Teramoto, Natural Gas Gathering Liquefaction and Ship Transportation for Thermal Power Genration, Journal of Cryogenics and Superconductivity Society of Japan, Vol. 32, No. 3, 1997 , pp 6-12.

[12] R. Moore and I. Long, A low-cost deepwater gas development solution using subsea dehydration, The APPEA journal, 56(2), p 604-604, 2016, https://doi.org/10.1071/AJ15110.

[13] Y. Bai and Q. Bai, Subsea engineering handbook. $2^{\text {nd }}$ ed, Gulf Professional Publishing, 2018.

[14] T. Saeki, J. Kameda, On the issues of economical feasibility (in Japanese), Methane Hydrate Forum, Presented in Tokyo, Japan, Nov. 2017. 